\title{
Ageism: we are our own worst enemy
}

\section{Introduction}

In 1969, Butler (1969) first coined the term "ageism" to confront "prejudice by one age group toward other age groups" (pp. 243). As with other "isms," such as racism and sexism, ageism leads to bigotry and discrimination, though it is a very distinct beast in that, for the most part, other "isms" refer to those different from ourselves: distinct, mutually exclusive, and impervious groups. Conversely, age is a fluid social construct in which we are all intimately bound as we move through the lifespan, transitioning in and out of different age-groups. Unlike other "isms," individuals negotiate shifts from the "in-group" of youth to the "out-group" of old age. Yet we are all immersed, largely unconsciously, in this ubiquitous but too often unrecognized "ism" that needs to be named and challenged.

Scientific evidence underscores the seriousness of ageism as a social issue. This was highlighted in a recent paper examining age stereotypes in social media (Levy et al., 2014a). Levy and colleagues found that $98.8 \%$ of Facebook group descriptions of older adults reflected negative stereotypes, with $74 \%$ of descriptions directly attacking older adults. For example, "...Old People ...are a burden on society. I hate everything about them..." (pp.173). What is of most concern is that these descriptors were not considered in breach of Facebook's policy on hate speech, which prohibits the singling out of individuals based on race, ethnicity, national origin, religion, sex, gender, sexual orientation, disease, or disability, but not age.

Ageism reflects our infatuation with youth and beauty that buffer our fears of the losses paired with "growing old," such as the loss of beauty, health, functioning, independence, usefulness, and ultimately, life. Palmore (2015) likens ageism to a "social disease" (pp. 874), that continues to contaminate generations through oversimplified representations of older adults in our culture, media, literature, and social discourse. These representations, or age stereotypes, are predominantly negative and can reduce older people to a homogenous group defined by their weakness, sickness, and senility.

Ageism is found in all walks of life, such as healthcare, the work-place, social policy, and everyday interactions, and can directly impact older adult's health and well-being. A full review is beyond the scope here and has been previously well covered (e.g. Robb et al., 2002; Bugental and Hehman, 2007; Finklestein and Farrell, 2007; Eymard and Douglas, 2012; North and Fiske, 2012; Anderson et al., 2013; North and Fiske, 2013). Instead, I want to focus on the lesser known, indirect effects of ageism. That is, how social and cultural norms reflected in ageism and age stereotypes impact upon our attitudes, expectations, and perceptions of our aging, often in harmful and self-fulfilling ways. In other words, have we become our own worst enemy?

\section{Impacts of ageism on health and well-being}

Attitudes and assumptions surrounding health and aging are heavily embedded within social cognitions. A consistent and normative assumption is that with age comes inevitable physical and cognitive decay (Golub and Langer, 2007). Golub and Langer argue such assumptions can lead to a "self-fulfilling prophecy of decline" (pp. 15). In a similar vein, Levy (2009) argues, in her Stereotype Embodiment theory, that social cognitions and age stereotypes become internalized and reinforced across the lifespan developing into beliefs about, expectations for, and self-perceptions of, one's own aging process. These negative attitudes toward aging are hypothesized to manifest into poor health outcomes through three pathways: psychological (through poor expectations regarding aging), physiological (for example, through suppression of the autonomic nervous system), and behavioral (reducing motivation to perform preventive health behaviors). There is strong evidence in support for this multiple pathway hypothesis. For instance, priming negative age stereotypes has been found to adversely affects older adults' performance in a number of domains including cognitive functioning and driving performance (Levy, 2003; Hess and Hinson, 2006), with this effect being maximized when the stereotype matches the task (Levy and Leifheit-Limson, 2009). Exposure to negative agestereotypes results in poorer subjective health, higher feelings of loneliness and more frequent dependency behaviors (Coudin and Alexopoulos, 
2010), and reduced will-to-live (Marques et al., 2014), in older adults. It has also been shown that holding negative age-stereotypes is associated with depression and poor well-being (Rothermund, 2005), increases the risk of hospitalization over a ten-year period (Levy et al., 2015), heightens cardiovascular response to stress (Levy et al., 2000), adversely influences recovery from cardiovascular events (Levy et al., 2006), and is even associated with greater evidence of Alzheimer's pathology such as higher hippocampal-volume loss and greater accumulation of neurofibrillary tangles and amyloid plaques (Levy et al., 2016).

Levy's (2009) theory, and others in this burgeoning field of research (e.g. Diehl's (2010) Awareness of Age-Related Change theory, and Montepare's (2009), subjective age and ageidentity work) have led to a body of work showing the numerous manifestations of internalized agestereotypes. Poor self-perceptions of aging have been shown to predict poor physical functioning and functional status (Levy et al., 2002), subjective health (Jang et al., 2004; Moor et al., 2006), cardiovascular health (Levy et al., 2009), and cognition (Robertson et al., 2016). In addition, a decline in self-perceptions of age have been linked to an increased risk in mortality in adults over 65 years of age over a 16-year period (Sargent-Cox et al., 2014). The impact of poorer expectations remain after controlling for other risk factors such as age, gender, physical health, cognitive functioning, and well-being (as in Sargent-Cox et al., 2014).

The helplessness that many older adults feel when it comes to aging and health is highlighted in a recent study of adults 80 years and over (Stewart et al., 2011). The authors found that participants who attributed causes of chronic illness to "old age" (i.e. heart disease, cancer, and diabetes) had significantly poorer outcomes including lower positive health behaviors (e.g. diet, exercise, rest), higher perceived health symptoms, and increased risk of mortality over two years. Of great interest, the participants of this study were more likely to rate the cause of their chronic illness to "old age" $(52.9 \%)$, than to unhealthy behaviors $(20.2 \%)$, providing support for the notion that, not only is the stereotype of the ill and helpless older person widespread, but that it self-perpetuates into maladaptive and harmful attitudes. As Stewart and colleagues conclude, the adage "to be old is to be ill" (pp.9) is alive and well.

Another way in which aging attitudes influence late life health and well-being is through behaviors and health beliefs. For example, stronger endorsement of negative age-stereotypes is associated with a higher likelihood of attributing health and health outcomes to an external locus of control (SargentCox and Anstey, 2015), an attribution process that is linked to poor health behaviors (Sarkisian et al., 2005; Gotz et al., 2011; Helmer et al., 2012; Scheffer et al., 2012). Wurm and colleagues (2013) examined the influence of self-perceptions of aging on self-regulation strategies in the face of a serious health event and found that those who had negative perceptions were less likely to use strategies that promote a healthy lifestyle and recovery.

Negative attitudes and perceptions of aging have also been shown to manifest as barriers to seeking timely healthcare treatment, particularly with conditions that are considered age related. For instance, older adults who attributed depression to aging, as opposed to illness, were more than four times likely to believe that depressive symptoms were not important to discuss with a doctor (Sarkisian et al., 2003).

The flip side of this phenomenon is that those who are able to maintain positive attitudes, expectations, and perceptions of their own aging are more likely to have better health and wellbeing outcomes in late life. Positive aging attitudes have been associated with better recovery from poor health events such as myocardial infarction (Levy et al., 2006) and severe disability (Levy et al., 2012) in older adults. Undertaking regular physical activity is more likely for middle-aged and older adults who hold more positive self-perceptions of aging (Wurm et al., 2010). In fact, the majority of studies that have examined the influence of perceptions of aging on health and longevity have found that more positive attitudes were associated with better health outcomes and higher longevity in older adults, with effect sizes ranging between 1.025 and 3.772 (Westerhof et al., 2014).

Of course, it could be argued that the direction of these associations are such that those in good health will naturally be able to maintain positive attitudes toward their own aging. However, there is evidence to suggest that, as Levy (2009) predicts, it is the attitudes toward aging that drive the outcome. For example, using longitudinal data to test directional hypotheses, we showed that the best-fitting model for adults 65 years and over was that which allowed self-perceptions of aging to predict change in physical functioning over time (measured as an objective physical performance battery), rather than the opposite direction (i.e. physical decline predicting selfperceptions of aging) (Sargent-Cox et al., 2012b). Further, these results remained after controlling for demographic and health factors known to impact on both self-perceptions of aging and physical functioning in older adults. In other words, positive self-perceptions of aging were shown to be 
protective against decline in physical functioning over a 16-year period.

In summary, there is a growing body of evidence indicating that cultural beliefs and stereotypes that are perpetuated and reinforced across the lifespan manifest as personal beliefs and attitudes regarding aging and the aging process. In turn, these personal beliefs and attitudes are impacting health and wellbeing outcomes in older adults, most likely through several pathways, such as cognitive, psychological, and behavioral. Of course, the question then becomes; if our beliefs about how we age are having serious consequences for how we actually age, what can we do to ensure that we have positive and productive aging attitudes?

\section{Combating ageism}

This brief overview of the literature highlights the need to address ageism not only at a societal level, but also at the individual level. This suggests different strategies are necessary; that is, addressing negative and overly simplified representations of older adults and the aging process, while also promoting positive personal views, attitudes, and expectations of growing older.

\section{Challenging and changing stereotypes}

Once formed, stereotypes have been shown to be resistant to change (Baron-Epel and Kaplan, 2001; Klein et al., 2005), though research is providing promising interventions, strategies, and techniques. A strategy that has been shown to be highly efficacious for improving attitudes to stereotyped groups is intergroup contact (Pettigrew, 2008). It is argued that ageism is perpetuated by societal segregation where children, young- and middleaged adults, as well as older adults are relegated to school, the workforce, and retirement or care, respectively, with limitations for contact and interaction between groups (Grefe, 2011). Quantity of contact between age groups (e.g. children and older adults) has been shown to improve implicit attitudes and associations, while quality of contact improves explicit attitudes (e.g. Tam et al., 2006). A limitation to intergroup contact is the availability of high quality and positive direct contact between groups. Recent research has overcome this constriction by investigating the efficacy of imagined contact in changing attitudes. Improved behavioral propensities (Turner and West, 2012), more projection of positive traits between groups (Stathi and Crisp, 2008), and increased confidence in successful future intergroup interactions (Stathi et al., 2011) have generally been shown in imagined contact research. More specifically, imagined contact with older adults has been shown to reduce implicit (Turner and Crisp, 2010) and explicit negative attitudes toward older adults, as well as inter age-group anxiety and bias (Turner et al., 2007).

Another well-researched strategy for reducing stereotypes is through an educational approach that aims to correct inaccurate beliefs and provide more precise information on the aged and aging through seminars, literature, and education programs. Mixed results have been found (e.g. Lohr et al., 1988; Fonesca et al., 2009; Samra et al., 2013); however, it is becoming a popular approach for including in health professional curriculums (Samra et al., 2013; Sarabia-Cobo and Pfeiffer, 2015).

\section{Improving attitudes toward own aging}

Challenging negative age-stereotypes and representing the heterogeneous experiences of aging in the media is an essential step in improving the health and well-being of all of us as we age. However, this is not an overnight fix and it is necessary to identify ways to improve individuals' attitudes and expectations of aging, particularly for older cohorts who have already internalized a lifetime of negative stereotypes. A recent metaanalysis showed that the effects of negative age-stereotypes on behaviors and performance in older adults are nearly three times larger than positive age-stereotypes (Meisner, 2012), highlighting the difficulty of simply offsetting negative age-stereotypes by boosting positive ones. Nonetheless, Levy et al. (2014b) have shown that strengthening positive stereotypes is possible. In adults 60 years and over, they found that an implicit positive age-stereotype intervention significantly strengthened positive age-stereotypes, weakened negative age-stereotypes, improved self-perceptions of aging, and over an eight-week period, improved physical functioning.

Intervention studies that have focused on selfperceptions of aging also provide hope that change is possible and meaningful. For example, two studies have shown that positive feedback can not only improve perceptions of age, but also physical performance (Stephan et al., 2013) and cognitive functioning (Miche and Wahl, 2013).

Another avenue of research examines the impact of education on older adults' attitudes. Providing information on the positive aspects of aging as well as correcting misconception and false beliefs can significantly improve middle-aged and older adults expectations and attitudes toward aging (Bardach et al., 2010; Wolff et al., 2014). Further, a randomized control trial showed that improvements 
in attitudes through an education intervention was also related to improvement in physical activity levels over time (Wolff et al., 2014).

The notion of imagined contact theory has also been used to investigate change in attitudes toward own aging. For example, imaging interaction with a 75-year old person was associated with more positive expectations of aging for male university students, compared to a control condition (Prior and Sargent-Cox, 2014). In particular, this study found that the relationship between imagined contact and expectation of aging was mediated by aging anxiety.

Another potential avenue for improving attitudes toward aging may be through fostering psychological resources that have been shown to protect or promote positive attitudes. Wurm and Benyamini (2014) found in adults aged between 40 years and 85 years, the relationship between negative selfperceptions of aging and poorer self-rated health, physical, and psychological health was moderated by optimism. In other words, those who had higher optimism had better health outcomes regardless of self-perception of aging scores. In another longitudinal study, the psychological resources of self-esteem and expectancy of control were shown to protect positive self-perceptions of aging in the face of declining physical functioning (SargentCox et al., 2012a). Similarly, high internal health locus of control have been found to be associated with lower endorsement of negative age-stereotypes (Sargent-Cox and Anstey, 2015). The major limitation to this research is the ambiguity regarding directionality of the relationships, but they do provide promise and a path for future research.

The goals of addressing age-stereotypes and challenging negative attitudes regarding the aging process are important and necessary components in tackling the numerous direct and indirect effects of ageism. Nevertheless, we are still a long way from understanding the best way to tackle these. As Kotter-Grühn (2015) points out, there remains a large gap in our knowledge. For instance, we need more research on prevention and intervention strategies to determine whether attitudes can be changed individually and at the broader societal level and the stability of effects of these interventions on change in attitudes and behaviors. We need to better understand between-person differences in the vulnerability of internalizing age stereotypes and the mechanisms that drive potential within-person changes in attitudes and self-stereotyping. We need to better understand who the most appropriate targets for interventions are, and how we can best reach those groups. And we need to decide how would we could implement and fund these programs.
However, all of this becomes a moot point until ageism itself is taken seriously. Until then, it is unlikely that the necessary knowledge will be gathered nor the necessary changes will take place. Until we really know that ageism is seriously affecting all of us and are prepared to invest resources necessary to tackle it, then we will continue to live in a society where negative views of aging go unchallenged: and as such, we all suffer the consequences.

\section{Where to from here?}

So where to from here? It is my belief that as professionals who work in the aging industry we need to take a leading role in challenging ageism. We already do this in a multitude of ways, through research, teaching, and our professional dealings every day. However, we can do more by naming ageism, challenging it, and bringing greater awareness to the repercussions.

We are embedded in an agist society, therefore we ourselves have been exposed to, and have internalized, agist beliefs to some degree. A crucial step in fighting ageism is firstly acknowledging and challenging our own attitudes and views about aging. As recently highlighted by John Beard, Director of the World Health Organization's Department of Ageing and Life Course, the majority of us are unaware of the age-stereotypes we subconsciously hold (World Health Organisation, 2016). Introspection has been shown to be efficacious in attitudinal change (Wilson and Hodges, 1992), and while stereotypes are often automatically activated, this activation can be moderated through a number of techniques including mindfulness (Djikic et al., 2008). Without acknowledging our own views, we are susceptible to confirmation bias, whereby new information we receive is interpreted within our established belief structure, thus perpetuating and deepening our views.

Another benefit of acknowledging and challenging our own agist attitudes is that we can then monitor our language and behavior. Language reflects our assumptions, judgments, and attitudes, whether implicitly or explicitly, and conveys these beliefs to others (Gendron et al., 2015). In fact, $\mathrm{Ng}$ (2007) argues that through our language we normalize prejudice and discrimination through verbal scripts that become part of everyday speech. For instance, the term "senior moment," a phrase that perpetuates the idea that to be old is to be cognitively incompetent, has become normalized and acceptable in our everyday lexicon (Bonnesen and Burgess, 2003).

A further step we can each take is to challenge and call out ageism, discrimination, and the 
perpetuation of age stereotypes around us. A good example comes from a recent paper by Pachana and colleagues (Pachana et al., 2015), who surveyed 157 researchers across Australia and the USA about ethics proposals for aging research. A major theme was that older populations were becoming the lost voices of research, with $53 \%$ of researchers citing problems gaining ethics due to consent and information requirements, and a further $37 \%$ lamenting concerns regarding assessing capacity and informed consent. Ethical review boards clustered aging research participants as particularly "vulnerable," raising concerns of stereotypical assumptions of older people's capacity to participate due to fatigue, distress, or an inability to recall information. Research such as this helps us understand the bias that has, with all good intention, crept into aging research and challenges researchers to address and change these attitudes.

\section{Conclusion}

Ageism is pervasive, widely accepted, and normative in many cultures and societies (e.g. Boduroglu et al., 2006). More importantly, ageism is a form of prejudice that goes unchallenged, and even celebrated in many fields, such as in the media, advertising, through jokes and everyday language. The direct consequences of ageism that results in discrimination and prejudice in a variety of fields including the workplace, social policy, and healthcare, are reasonably well known and largely acknowledged. These consequences are multifaceted, multidimensional, and complicated, and I by no means suggest that being mindful and naming ageism and prejudice is a panacea to the problem. However, gaining insight and understanding into the more insidious indirect effects of ageism is an important step in improving our own aging process, and creating the impetus for us to be more proactive in research and action for challenge and change.

\section{Conflict of interest}

None.

\section{Kerry SARgent-Cox}

Centre for Research on Ageing, Health and Wellbeing, Research School of Population Health, Australian National University, Canberra, ACT, 0200, Australia Email: Kerry.Sargent-Cox@anu.edu.au.

\section{References}

Anderson, K. A., Richardson, V. E., Fields, N. L. and Harootyan, R. A. (2013). Inclusion or exclusion?
Exploring barriers to emplyment for low-income older adults. Fournal of Gerontological Social Work, 56, 318-334. doi:10.1080/01634372.2013.

Bardach, S. H., Gayer, C. C., Clinkinbeard, T., Zanjani, F. and Watkins, J. F. (2010). The malleability of possible selves and expectations regarding aging. Educational Gerontology, 36, 407-424. doi:10.1080/03601270903212393.

Baron-Epel, O. and Kaplan, G. (2001). General subjective health status or age-related subjective health status: does it make a difference? Social Science and Medicine, 53, 1373-1381.

Boduroglu, A., Yoon, C., Luo, T. and Park, D. C. (2006). Age-related stereotypes: a comparison of American and Chinese cultures. Gerontology, 52, 324-333. doi: $10.1159 / 000097614$.

Bonnesen, J. L. and Burgess, E. O. (2003). Senior moments: the acceptability of an ageist phrase. Fournal of Aging Studies, 18, 123-142. doi:10.1016/j.jaging.2004.01.008.

Bugental, D. B. and Hehman, J. A. (2007). Ageism: a review of research and policy implications. Social Issues and Policy Review, 1, 173-216. doi:10.1111/j.1751-2409.2007.00007.x.

Butler, R. N. (1969). Age-ism: another form of bigotry. The Gerontologist, 9, 243-246. doi:10.1093/geront/9.4_Part_1.243.

Coudin, G. and Alexopoulos, T. (2010). 'Help me! I'm old!' How negative aging stereotypes create dependency in older adults. Aging and Mental Health, 14, 516523.

Diehl, M. (2010). Awareness of age-related change: examination of a (mostly) unexplored concept. Fournal of Gerontology; Social Sciences, 65B, 340-350.

Djikic, M., Langer, E. J. and Stapleton, S. F. (2008). Reducing stereotyping through mindfulness: effects on automatic stereotype-activated behaviors. Fournal of Adult Development, 15, 16-111. doi:10.1007/s10804-008-9040-0.

Eymard, A. S. and Douglas, D. H. (2012). Ageism among health care providers and interventions to improve their attitudes toward older adults: an integrative review. Fournal of Gerontological Nursing, 38, 26-35. doi:10.3928/00989134-20120307-09.

Finklestein, L. M. and Farrell, S. K. (2007). An expanded view of age bias in the workplace. In K. S. Shultz and G. A. Adams (eds.), Ageing and Work in the 21st Century (pp. 73-108). Mahwah, NJ: Lawrence Erlbaum Asc.

Fonesca, A., Goncalves, D. and Martin, I. (2009). Changing attitudes towards ageing and the aged amongst psychology students. European Fournal of Education, 44, 455-466. doi:10.1111/j.1465-3435.2009.01396.x.

Gendron, T. L., Welleford, E. A., Inker, J. and White, J. T. (2015). The language of ageism: why we need to use words carefully. The Gerontologist, 1-10. doi:10.1093/geront/gnv066.

Golub, S. A. and Langer, E. J. (2007). Challenging assumptions about adult development: implications for the health of older adults. In C. M. Aldwin, C. L. Park and A. Spiro III (eds.), Handbook of Health Psychology and Aging (pp. 9-29). New York: The Guildford Press.

Gotz, M., Hapke, U. and Lampert, T. (2011). Health locus of control and health behavior: results from a nationally 
representative survey. Psychology, Health and Medicine, 16, 129-140. doi:10.1080/13548506.2010.521570.

Grefe, D. (2011). Combating ageism with narrative and intergroup contact: possibilities of intergenerational connections. Pastoral Psychology, 60, 99-105. doi:10.1007/s11089-010-0280-0.

Helmer, S. M., Kramer, A. and Mikolajcz, R. T. (2012). Health-related locus of control and health behavior among university students in North Rhine, Westphalia, Germany. BMC Research Notes, 5, 703. doi:10.1186/1756-0500-5-703.

Hess, T. M. and Hinson, J. T. (2006). Age-related variation in the influences of aging stereotypes on memory in adulthood. Psychology \& Aging, 21, 621-625.

Jang, Y., Poon, L. W. and Martin, P. (2004). Individual differences in the effects of disease and disability on depressive symptoms: the role of age and subjective health. International fournal of Aging E Human Development, 59, 125-137. doi:10.2190/RT1W-2HD7-KG5X-K1FB.

Klein, D. A., Council, K. J. and McGuire, S. L. (2005). Education to promote postive attitudes about aging. Educational Gerontology, 31, 591-601. doi: $10.1080 / 30601270591003355$.

Kotter-Gruhn, D. (2015). Changing negative views on aging: implications for intervention and translational research. Annual Review of Gerontology \& Geriatrics, 35, 167. doi:org/10.1891/0198-8794.35.167.

Levy, B. (2003). Mind matters: cognitive and physical effects of aging self-stereotypes. The fournals of Gerontology, 58B, P203-P211. doi:10.1093/geronb/58.4.P203.

Levy, B. (2009). Stereotype embodiment: a psychosocial approach to aging. Current Psychological Directions in Science, 18, 332-336. doi:10.1111/j.1467-8721.2009.01662.x.

Levy, B., Chung, P. H., Bedford, T. and Navrazhina, K. (2014a). Facebook as a site for negative age stereotypes. The Gerontologist, 54, 172-176. doi:10.1093/geront/gns 194.

Levy, B., Ferrucci, L., Zonderman, A. B., Slade, M. D., Troncoso, J. and Resnick, S. M. (2016). A culture-brain link: negative age stereotypes predict Alzheimer's disease biomarkers. Psychology $\mathcal{E}$ Aging, 31, 82. doi: $10.1037 /$ pag0000062.

Levy, B., Haursdorff, J. M., Hencke, R. and Wei, J. Y. (2000). Reducing cardiovascular stress with positive self-stereotypes of aging. Fournal of Gerontology: Psychological Sciences, 55B, P205-P213. doi:10.1093/geronb/55.4.P205.

Levy, B. and Leifheit-Limson, E. (2009). The stereotype-matching effect: greater influence on functioning when age steretoypes correspond to outcomes. The fournals of Gerontology: Series B Psychological Science Social Science, 55B, P205-P213. doi:10.1093/geronb/55.4.P205.

Levy, B., Pilver, C., Chung, P. H. and Slade, M. D. (2014b). Subliminal strengthening: improving older individuals physical function over time with an implict-age-stereotype intervention. Psychological Science, 25, 2127-2135. doi:10.1177/0956797614551970.

Levy, B., Slade, M., May, J. and Caracciolo, E. (2006). Physical recovery after acuty myocardial infarction: positive age self-stereotypes as a resource. International fournal of Aging \& Human Development, 62, 285-301. doi:10.2190/EJK1-1Q0D-LHGE-7A35.
Levy, B., Slade, M. D., Chung, P. H. and Gill, T. M. (2015). Resliency over time of elders' age stereotypes after encountering stressful events. Fournals of Gerontology: $B$ Psychological and Social Sciences, 70, 886-890. doi:10.1093/geronb/gbu082.

Levy, B., Slade, M. D. and Kas1, S. V. (2002). Longitudinal benefits of positive self-perception of aging on functional health. The fournals of Gerontology, 57, P409-P417. doi:10.1093/geronb/57.5.P409.

Levy, B., Slade, M. D., Murphy, T. E. and Gill, T. M. (2012). Association between positive age stereotypes and recovery from disability in older persons. $\mathcal{f} A M A, 308$, 1972-1973. doi:10.1001/jama.2012.14541.

Levy, B., Zonderman, A., Slade, M. and Ferrucci, L. (2009). Age stereotypes held earlier in life predict cardiovascular events in later life. Psychological Science, 20, 296-298.

Lohr, M. J., Essex, M. J. and Klein, M. H. (1988). The relationships of coping responses to physical health status and life satisfaction among older women. Fournal of Gerontology, 43, P54-P60. Retrieved from http://E:/PhD/Journal articles/Lohr,Essex\&Klein,1988.doc.

Marques, S., Lima, M. L., Abrams, D. and Swift, $\mathbf{H}$. (2014). Will to live in older people's medical decisions: immediate and delayed effects of aging stereotypes. Fournal of Applied Social Psychology, 44, 399-408. doi:10.1111/jasp. 12231.

Meisner, B. A. (2012). A meta-analysis of positive and negative age stereotype priming effects on behavior among older adults. Fournals of Gerontology: B Psychological and Social Sciences, 67, 13-17. doi:10.1093/geronb/gbr062.

Miche, M. and Wahl, H.-W. (2013). "Its because of my age." The influence of experimentally increased salience of age-related changes in cogntive functioning on self-perceptions of ageing. Paper presented at the 66th Annual Sientific Meeting of the Gerontological Society of American, New Orleans, LA.

Montepare, J. M. (2009). Subjective Age: toward a guiding lifespan framework. International fournal of Behavioral Development, 33, 42-46. doi:10.1177/0165025408095551.

Moor, C., Zimprich, D., Schmitt, M. and Kliegel, M. (2006). Personality, aging self-perceptions and subjective health: a mediation model. International fournal of Aging $\mathcal{E}$ Human Development, 63, 241.

doi:10.2190/AKRY-UM4K-PB1V-PBHF.

Ng, S. H. (2007). Language-based discrimination: blatant and sublte forms. Fournal of Language and Social Psychology, 26, 106-122. doi:10.1177/0261927X07300074.

North, M. S. and Fiske, S. T. (2012). An inconvenienced youth? Ageism and its potential intergenerational roots. Psychological Bulletin, 138, 982-997. doi: $10.1037 / \mathrm{a} 0027843$.

North, M. S. and Fiske, S. T. (2013). Subtyping agesim: policy issues in succession and consumption. Social Issues and Policy, 7, 36-57. doi:10.1111/j.1751-2409.2012.01042.x.

Pachana, N. A., Liddle, J., Peel, N. M., Beattie, E., Juang, C. and Knight, B. G. (2015). Can we do better? Researchers experiences with ethical review boards on projects with later life focus. Fournal of Alzheimer's Disease, 43, 701-707. doi:10.3233/JAD-141956. 
Palmore, E. (2015). Ageism comes of age. Fournal of Gerontology: B Psychological and Social Sciences, 70, 873-875. doi:10.1093/geronb/gbv079.

Pettigrew, T. F. (2008). Future directions for intergroup contact theory and research. International fournal of Intercultural Relations, 32, 187-199. doi:10.1016/j.ijintrel.2007.12.002.

Prior, K. and Sargent-Cox, K. (2014). Student's expectations of ageing: an evaluation of the impact of imagined intergenerational contact and the mediating role of ageing anxiety. Fournal of Experimental Social Psychology, 55, 99-104. doi:10.1016/j.jesp.2014.06.001.

Robb, C., Chen, H. and Haley, W. E. (2002). Ageism in mental health adn health care: a critical review. fournal of Clinical Gerypsychology, 8, 1-12.

Robertson, D. A., King-Kallimanis, B. and Kenny, R. A. (2016). Negative perceptions of aging predict longituidnal decline in cognitive function. Psychology $\&$ Aging, 31, 71-81. doi:10.1037/pag0000061.

Rothermund, K. (2005). Effects of age stereotypes on self-views and adaptation. In W. Greve, K. Rothermund and D. Wentura (eds.), The Adaptive Self. Personal Continuity and Intentional Self-development (pp. 223-242). Göttingen, Germany: Hogrefe.

Samra, R., Griffiths, A., Cox, T. and Conroy, S. (2013). Changes in medical student and doctor attitudes toward older aduls after an intervention: a systematic review. fournal of American Geriatrics Society, 61, 1188-1196. doi:10.1111/jgs.12312.

Sarabia-Cobo, C. M. and Pfeiffer, C. C. (2015). Changing negative stereotypes regarding ageing in undergraduate nursing students. Nurse Education Today, 35, e60-e64. doi:10.1016/j.nedt.2015.06.006.

Sargent-Cox, K. and Anstey, K. J. (2015). The relationship between age-steretoypes and health locus of control across adult age-groups. Psychology and Health, 30, 652-670. doi:10.1080/08870446.2014.974603.

Sargent-Cox, K., Anstey, K. J. and Luszcz, M. A. (2012a). Change in health and self-perceptions of ageing over 16 years: the role of psychological resources. Health Psychology, 31, 423-432. doi:10.1037/a0027464.

Sargent-Cox, K., Anstey, K. J. and Luszcz, M. A. (2012b). The relationship between change in self-perceptions of aging and physical functioning in older adults. Psychology and Aging, 27, 750.

Sargent-Cox, K., Anstey, K. J. and Luszcz, M. A. (2014). Longitudinal change of self-perceptions of aging and mortality. Fournals of Gerontology: Psychological Science, 69, 168-173. doi:10.1093/geronb/gbt005.

Sarkisian, C. A., Lee-Henderson, M. H. and Mangione, C. M. (2003). Do depressed older adults who attribute depression to "old age" believe it is important to seek care? fournal of General Internal Medicine, 18, 10011005.

Sarkisian, C. A., Prohaska, T. R., Wong, M. D., Hirsch, S. H. and Mangione, C. M. (2005). The relationship between expectations of aging and physical activity among older adults. Fournal of Internal Medicine, 20, 911-915. doi:10.1111/j.1525-1497.2005.0204.x.

Scheffer, C. et al. (2012). Delay discounting, locus of controland cognitive impulsiveness independelty predict tobacco dependence treatment outcomes ina a highly dependent, lower socioeconomic group of smokers. The American fournal of Addictions, 21, 221-232. doi:10.1111/j.1521-0391.2012.00224.x.

Stathi, S. and Crisp, R. J. (2008). Imagining intergroup contact promote projection to outgroups. Fournal of Experimental Social Psychology, 44, 943-957. doi:10.1016/j.jesp.2008.02.003.

Stathi, S., Crisp, R. J. and Hogg, M. A. (2011). Imagining intergroup contact enables member-to-group generalization. Group Dynamics: Theory, Research, and Practice, 15, 275-284. doi:10.1037/a0023752.

Stephan, Y., Chalabaev, A., Kotter-Grühn, D. and Jaconelli, A. (2013). "Feeling younger, being stronger": an experimental study of subjective age and physical functioning among older adults. Fournals of Gerontology: $B$ Psychological and Social Sciences, 68, 1-7. doi: 10.1093/geronb/gbs037.

Stewart, T. L., Chipperfield, J. G., Perry, R. P. and Weiner, B. (2011). Attributing illness to 'old age:' consequences of a self-directed stereotype for health and mortality. Psychology and Health, iFirst, 1-17. doi:10.1080/08870446.2011.630735.

Tam, T., Hewstone, M., Harwood, J., Voci, A. and Kenworthy, J. (2006). Intergroup contact and grandparent-grandchild communication: the effects of self-disclosure on implicit and explicit biases against older people. Group Processes and Intergroup Relations, 9, 413-429. doi:10.1177/1368430206064642.

Turner, R. N. and Crisp, R. J. (2010). Imagining intergroup contact reduces implicit prejudice. British fournal of Social Psychology, 49, 129-142. doi:10.1348/014466609X419901.

Turner, R. N., Crisp, R. J. and Lambert, E. (2007). Imagining intergroup contact can improve intergroup attitudes. Group Processes and Intergroup Relations, 10, 427-441. doi:10.1177/1368430207081533.

Turner, R. N. and West, K. (2012). Behavioral consequences of imaging intergroup contact with stgmatized outgroups. Group Processes and Intergroup Relations, 15, 193-202. doi:10.1177/1368430211418699.

Westerhof, G. J. et al. (2014). The influence of subjective aging on health and longevity: a meta-analysis of longitudinal data. Psychology \& Aging, 29, 793802.

Wilson, T. D. and Hodges, S. D. (1992). Attitudes as temporary constructions. In L. L. Martin and A. Tesser (eds.), The Construction of Social fudgements (pp. 37-66). Hillsdale, New Jersey: Lawrence Erlbaum Associates.

Wolff, J. K., Warner, L. M., Ziegelmann, J. P. and Wurm, S. (2014). What do targeting positive views on ageing add to a physical activity intervention in older adults? Results from a randomised controlled trial. Psychology $\mathcal{E}$ Health, 29, 915-932. doi:10.1080/08870446.2014.896464.

World Health Organisation. (2016). Discrimination and negative attitudes about ageing are bad for your health [Press release]. Available at: http:/www.who.int/mediacentre/news/releases/2016/ discrimination-ageing-youth/en/.

Wurm, S. and Benyamini, Y. (2014). Optimism buffers the detrimental effect of negative self-perceptions of ageing on physical and mental health. Psychology $\mathcal{E}$ Health, 29, 832-848. doi:10.1080/08870446.2014.891737. 
Wurm, S., Tomasik, M. J. and Tesch-Römer, C. (2010).

On the importance of a positive view on ageing for physical exercise among middle-aged and older adults:

cross-sectional and longitudinal findings. Psychology $\mathbb{F}$

Health, 25, 25-42. doi:10.1080/08870440802311314.
Wurm, S., Warner, L. M., Ziegelmann, J. P., Wolff, J. K. and Schuz, B. E. C. (2013). How do negative self-perceptions of aging become a self-fullfilling prophecy?. Psychology \& Aging, 28, 10881097. doi:10.1037/a0032845. 